\title{
A comprehensive assessment of automatic logistic regression model selection methods
}

\section{Louis Ehwerhemuepha ( $\nabla$ lehwerhemuepha@choc.org)}

Children's Hospital of Orange County https://orcid.org/0000-0003-1369-7954

Cyril Rakovski

Chapman University

Research article

Keywords: Model performance, model selection, effect size estimation and direction

Posted Date: November 7th, 2019

DOI: https://doi.org/10.21203/rs.2.16960/v1

License: (9) This work is licensed under a Creative Commons Attribution 4.0 International License.

Read Full License 


\title{
A comprehensive assessment of automatic logistic regression model selection methods
}

\author{
Louis Ehwerhemuepha ${ }^{\mathrm{a}, \mathrm{b}}$ and Cyril Rakovski ${ }^{\mathrm{b}}$ \\ ${ }^{\mathrm{a}}$ School of Computational and Data Science, Chapman University, Orange, CA, USA; ${ }^{\mathrm{b}} \mathrm{CHOC}$ \\ Children's Hospital, Orange, CA, USA
}

ARTICLE HISTORY

Compiled October 21, 2019

\begin{abstract}
Background: Current studies of variable selection methods are based on small datasets for which the true underlying predictors and corresponding effect sizes may not be known precisely. We designed and implemented a large-scale simulation study to assess the ability of stepwise logistic regression variable selection and lasso regression methods to identify the correct underlying model.

Methods: We simulated data based on a random multivariate distribution over an extensive range of covariate correlation structures, sample sizes, number of covariates, number of true predictors, and effect sizes. We considered correlation structures reflecting the scenarios of independence across all covariates; two low correlation blocks and a block of zero correlation; three blocks of high correlation, low correlation and zero correlation; and two blocks of high correlation with low cross block correlation and a block of zero correlation respectively.

Results: Our results show that the performances of stepwise regression, lasso regression, and random forest algorithm (as measured by the AUC, detection of true predictors, selection of noise variables, and effect size estimation) are affected by the number of true predictor variables, the correlation between the variables in the dataset, and the sample size. The number of noise variables in the dataset, although significant in some cases, has only a marginal effect on a variety of model performance statistic. Our results show that stepwise regression procedures are more likely to capture a true predictor, more likely to correctly estimate the true effect sizes, and more likely to have lower false discovery rates. Moreover, stepwise model selection based on the BIC criteria obtained the highest average AUC (0.7515) closely followed by lasso regression, stepwise model selection based on the AIC criteria, ridge regression, and random forest algorithm.

Conclusions: Stepwise procedures for variable selection are robust in the selection of the true underly predictor variables, estimation of corresponding effect sizes, and predictive model performance. Recommendations suggesting that stepwise procedures should be abandoned may be misguided.
\end{abstract}

\section{KEYWORDS}

Model performance; model selection; effect size estimation and direction

\section{Background}

Logistic regression is an integral part of data analysis focused on exploring the strength and direction of associations between a dichotomous response variable and a set of potential predictors, and is widely used by researchers [1], [2], [3], [4], [5], [6]. In general, the goal of these research studies is to identify the set of true predictors that are incorporated in the final model which is used for classification, prediction or interpretation. 
In the scenarios where the number of variables and consequently the number of possible models is small, exhaustive model building approach is computationally feasible. However, in this era of big data, large sample sizes are usually available [7], [8], [9] but also, many/all potential covariates are measured on every subject in the study which necessitates the use of automated model building techniques. There are several well-known automated model building techniques, stepwise regression procedures, best subsets regression, and shrinkage methods such as least absolute shrinkage and selection operator (lasso) and ridge regressions [10]. These methods can be combined with a model selection approaches such as statistical significance based on likelihood ratio, Wald or Analysis of Variance (ANOVA) tests or penalized likelihood statistics such as Aikaike Information Criteria (AIC), Bayesian Information Criteria (BIC) and Corrected Aikaike Information Criteria (AICC). In this study, we implement stepwise logistic regression with forward selection and backward elimination and the AIC and BIC. In forward selection, we start with a base (intercept-only) model and selectively add covariates one at a time given that such addition would lead to a better fit and stopping when it results in a poorer fit. The reverse is the case for backward elimination. We start with the full model (model containing all covariates) and selectively remove covariates one at a time only if such removal would lead to a better fit and stopping otherwise.

Forward selection with backward elimination is a combination of both forward and backward elimination but covariates previously removed from the model are given opportunities to re-enter the model based on additional constraints. These methods have not been exhaustively assessed. Known criticisms of stepwise regression procedure includes arguments that the regression coefficients of the final model chosen are too large and require shrinkage [10]; the corresponding confidence intervals for effects and predicted values are falsely narrow [11]; and the sample size is of little practical importance in determining the number of authentic variables contained in the final model [12]. Furthermore, the degree of correlation between the predictor variables influences the frequency with which authentic predictor variables found their way into the final model [12]; the R-squared value of the resulting model and significance of the covariates are inflated $[13,14]$. In a similar way, there have been criticisms of the shrinkage method. In particular, a previous study suggests that there are potential pitfalls arising with lasso estimation and that a significant portion of the parameter space of Lasso has higher mean-squared error than ordinary least squares algorithm for regression [15]. Several generalizations of the lasso have been developed such as the grouped lasso, elastic net, and adaptive lasso [16]. Furthermore, attempts have been made to quantify the false discovery rate of the lasso variable selection algorithm and researchers have developed a relationship between the shrinkage parameter and the false discovery rate [17].

Further, we estimate the effects of the number of true predictor and noise variables in a dataset, the correlation structure among the entire set of covariates, and the sample size on the performance of stepwise and lasso regression algorithms. We compared the algorithms classification accuracy through the area under the receiver operator characteristic curve (AUC) statistic, the correctness of the final model through the true and false variable discovery rates, and the precision of the estimated effect sizes in terms of their magnitudes and directions. We designed and implemented a large scale simulation study under an extensive range of parameter-defined scenarios that reflected real data structures, sizes and hypothetical true models. In particular, each simulation scenario was defined by a combination of the following parameters, effect sizes, total number of true predictor variables, total number of noise variables, and complex block correlation structure. 


\section{Methods}

In recent years, a large number of attributes are recorded/collected on each subject, and achieving the goal of finding the subset of variables associated with the variable of interest necessitates automated model building procedures due to the sheer number of competing models. Several methods such as stepwise regression, best subset selection and lasso regression have been developed and implemented in widely used statistical software [18, 19]. In our study, we focus on large scale simulations designed to assess the performance of stepwise variable selection for logistic regression, lasso regression, and random forest algorithm.

\section{Simulation Design}

we carried out a comprehensive simulations study over a range of common scenarios defined by number of true predictors, number of noise variables, effect sizes, correlation structure of the complete set of covariates, and sample sizes. In particular, we simulated the datasets used for this study using four correlation structures. The first, $R_{1}$, consists of 3 independence block correlation matrices where $k, l$, and $m$ represents the size of the corresponding block matrices. The second, $R_{2}$, consists of two low correlation block matrices and an independence block matrix to represent the scenario where there is low correlation between the columns of data. The third, $R_{3}$, consists of a block of high, low, and independence block correlation matrices to represent scenario where the columns of data consists of a set of high, low, and independent correlation. The fourth, $R_{4}$, is a more complex correlation structure incorporating a crossblock correlation structure with the highest correlation of all four correlation matrices.

$$
\begin{aligned}
R_{1} & =\left(\begin{array}{ccc}
I_{k} & 0 & 0 \\
0 & I_{l} & 0 \\
0 & 0 & I_{m}
\end{array}\right), R_{2}=\left(\begin{array}{ccc}
L_{k} & 0 & 0 \\
0 & L_{l} & 0 \\
0 & 0 & I_{m}
\end{array}\right), \\
R_{3} & =\left(\begin{array}{ccc}
H_{k} & 0 & 0 \\
0 & L_{l} & 0 \\
0 & 0 & I_{m}
\end{array}\right), R_{4}=\left(\begin{array}{ccc}
H_{k} & L_{k l} & 0 \\
L_{l k} & H_{l} & 0 \\
0 & 0 & I_{m}
\end{array}\right),
\end{aligned}
$$

We imposed a compound symmetry structure on the low and high correlation diagonal blocks $L_{k}$ and $H_{k}$ with off-diagonal values fixed at 0.2 and 0.6 respectively,

$$
L_{k}=\left(\begin{array}{cccc}
1 & 0.2 & \cdots & 0.2 \\
0.2 & 1 & \cdots & 0.2 \\
\vdots & \vdots & \ddots & \vdots \\
0.2 & 0.2 & \cdots & 1
\end{array}\right), H_{k}=\left(\begin{array}{cccc}
1 & 0.6 & \cdots & 0.6 \\
0.6 & 1 & \cdots & 0.6 \\
\vdots & \vdots & \ddots & \vdots \\
0.6 & 0.6 & \cdots & 1
\end{array}\right)
$$

and imposed equal correlation structure on the off-diagonal low correlation block $L_{k l}$ with all matrix values fixed at 0.2 ,

$$
L_{k l}=\left(\begin{array}{cccc}
0.2 & 0.2 & \cdots & 0.2 \\
0.2 & 0.2 & \cdots & 0.2 \\
\vdots & \vdots & \ldots & \vdots \\
0.2 & 0.2 & \ldots & 0.2
\end{array}\right)
$$


The indices $k, l$, and $m$ which denote the sizes of the blocks were set at three values of $(3,4,8),(3,8,12)$, and $(4,8,20)$ respectively. This implies that we considered 27 distinct correlation matrices, with the minimum number of variables set at 10 , and maximum at 40 .

We set the number of true predictor variables at $(2,5,10)$ with sample sizes of 1000 and 5000 . We set the corresponding effect sizes to values corresponding to small, medium, and large effect sizes of $\left(\log \left(\frac{1}{\sqrt{2}}\right), \log \sqrt{2}, \log 2\right)$ from which we randomly sampled with replacement. Lastly, we generated a binary response variable using a random binomial number generator with the probability of 1 set to the logistic function of the dot product of the effect sizes of the true predictor variables and the values of the variables.

We considered 648 distinct simulation scenarios corresponding to four correlation structures (with 27 distinct settings each), three values for the number of true predictors in the data, and two sample sizes. For each simulation scenario, we generate 1000 data sets (an overall total of 648,000 data sets) and applied six variable or model selection algorithms corresponding to stepwise reduction of AIC, stepwise reduction of BIC, lasso regression, ridge regression, another elastic net algorithm, and random forest. We averaged the model performance across the 1000 datasets for each scenario. The model performance metrics we captured includes the area under the operator receiver curve (AUC), proportion of true and false positive predictor variables in the model, proportion of true and false negative variables excluded from the model, the percentage difference in true effect sizes across the true predictor variables, and proportion of times the effect sizes were inflated, deflated, or within $10 \%$ of the true effect size. Furthermore, we kept track of the number of times the variable selection algorithms captured all true predictors without noise, and all true predictors with some noise. Using linear regression on the 648 simulation scenarios, we analyzed the effect of the number of true predictors, the number of noise variables, the correlation structure, and the sample size on the model performance statistics. 


\section{Results}

\section{Area under the receiver-operator characteristic curve}

Our results (Figure 1) suggest that averaged over all simulation scenarios, stepwise model selection by the BIC criteria produces the highest AUC $(0.7515)$, followed closely by lasso regression (0.7514), elastic net (0.7513), stepwise model selection by the AIC criteria (0.7496), ridge regression (0.7450), and random forest (0.7157).

\section{True and false positive predictors identified by the models}

Both stepwise and lasso regression tend to capture all the true predictors, and in Figure 2, we see that lasso regression is tends to capture all the true predictors in the dataset but at the cost of a significantly higher false discovery rate. This is followed by stepwise model selection using the AIC criteria with higher true discovery rate if the $\mathrm{p}$-values are ignored. This indicates that there are occasions where stepwise reduction of AIC captures a true predictor with $\mathrm{p}$-value $<0.05$, but ignoring the $\mathrm{p}$-value leads to significantly higher false discovery rate. On the other hand, stepwise reduction of BIC which tends to produce the most parsimonious model, tend to capture more than $99 \%$ of the true predictors in the dataset while possessing a significantly lower false discovery rate than other variable selection algorithm. This result seems to imply that for inference, stepwise regression may be preferred to the other variable selection algorithm to protect against false discoveries and the corresponding cost of decision making on false discoveries. However, we must note that there are research work on the selection of shrinkage parameter to help reduce the false discovery rate of lasso regression.

\section{Effect sizes}

The effect sizes obtained using stepwise regression procedures have been criticized as imprecise for the purpose of inference under the suspicion that the effect sizes are inflated, and that there is no way to adjust for multiple comparison. In this study, we defined the model derived effect sizes as normal if within $10 \%$ of the true effect size, otherwise as inflated or deflated depending on the direction of change. In Figure 4, we compare stepwise regression effect sizes to those of Lasso and Ridge regression. Our results indicate that Lasso and Ridge regression are more likely to produce effect sizes that are deflated (smaller) than the true effect sizes. The stepwise regression methods are more likely to inflate the effect sizes but on the average are more likely to produce effect sizes that are normal (within $10 \%$ of the true effect sizes) than both Lasso and Ridge regression. Lasso and Ridge regression are more likely to result in deflated effect sizes (between $70 \%$ and $100 \%$ of the true predictors) than normal effect sizes. On the other hand, the stepwise regression procedures are more likely to result in normal effect sizes (between $48 \%$ and $50 \%$ of the true predictors) than inflated or deflated effect sizes. In other words, while the effect sizes from Lasso and Ridge regression are more likely to be deflated, stepwise regression is more likely to produce the correct effect sizes. But between $28 \%$ and $30 \%$ of the effect sizes from stepwise regression will be inflated. In other words, although stepwise regression is more likely to inflate the effect sizes it is at this same time more accurate and precise than corresponding estimates from Lasso and Ridge regression. In addition, using the BIC as the model discrimination statistic may result in more accurate estimates of the true effect sizes than using the AIC statistic. 
Table 1. Regression analysis of factors associated with the AUC

\begin{tabular}{ccccccc}
\hline Variables & StepAIC & StepBIC & Lasso & Ridge & Elastic Net & Random Forest \\
\hline npreds & 0.0198 & 0.0190 & 0.0194 & 0.0198 & 0.0194 & 0.0176 \\
noise & -0.0003 & $N S$ & $N S$ & -0.0005 & -0.0001 & -0.0004 \\
typeR2 & 0.0048 & 0.0051 & 0.0050 & 0.0035 & 0.0049 & 0.0088 \\
typeR3 & 0.0075 & 0.0070 & 0.0071 & 0.0051 & 0.0067 & 0.0183 \\
typeR4 & 0.0051 & 0.0050 & 0.0049 & 0.0030 & 0.0047 & 0.0111 \\
ntrain5000 & 0.0095 & 0.0087 & 0.0078 & 0.0146 & 0.0079 & 0.0205 \\
\hline
\end{tabular}

Table 2. Regression summary of factors associated with the number of true positive predictors identified by the models

\begin{tabular}{ccccc}
\hline Variables & StepAIC & StepBIC & Lasso & Elastic Net \\
\hline npreds & -0.0079 & -0.0149 & -0.0036 & -0.0032 \\
noise & $N S$ & $N S$ & -0.0007 & -0.0006 \\
typeR2 & $N S$ & $N S$ & $N S$ & $N S$ \\
typeR3 & -0.0374 & -0.0610 & -0.0253 & -0.0231 \\
typeR4 & -0.0196 & -0.0319 & -0.0135 & -0.0124 \\
ntrain5000 & 0.0603 & 0.1160 & 0.0335 & 0.0298 \\
\hline
\end{tabular}

\section{Factors Associated with Model performance}

The AUC obtained by the variable and model selection algorithms increases as the sample size and number of true predictor variables increases. Furthermore, as the average correlation between all variables increases, the AUC increases as well. Expectedly, the AUC decreases as the number of noise variables in the dataset increases although not significant for stepwise reduction of BIC and Lasso regression. The effect of the number of noise variables on model performance of the other model/variable selection algorithm, measured by the AUC, is small with effect sizes in the $10^{-4}$ order of magnitude. Details are presented in Table 1 .

The negligible effect size $\left(\times 10^{-4}\right)$ of the number of noise variables imply that the AUC of predictive models are quite robust against the inclusion of noise variables. Based on the effect size of the number of true predictors and the number of noise variables, we can infer that including as many variables as possible prior to model development is to be encouraged. Consequently, it is desirable to collect variables as many variables as possible without negative implications with respect to the AUC. Furthermore, it is quite interesting to note that correlations between the predictor variables may help improve the AUC of the model. This is to be expected if some noise variables have high correlation with true predictor variables. In this case, the correlated noise variable may act as a proxy to a true predictor unknown to the investigator. Lastly, it goes without reason that the large the sample size, the higher the AUC of the model as the precision of parameter estimation is improved.

The number of true predictors identified by the final models increases with sample size but decreases with an increase in the average correlation among all variables, higher number of noise variables, and higher number of true predictor variables. However, the effect size of the number of noise variables is quite negligible $\left(\times 10^{-4}\right)$. This results are in line with the assertion that correlation among variables in the data may improve the AUC of the resulting model through capture of true predictors or a false predictors correlated with them even though it constitutes a false negative variable finding. The effect size of the sample size variable () indicates that the problem of capturing the true predictor variables may be improved in large sample settings. See Table 2 for details.

The number of noise variables and average correlation among all variables in the dataset results in reduced odds of selecting true negative variables. The higher the sample size, the lower the odds of selecting false negative variables. The regression coefficients indicate that 
Table 3. Regression summary of the factors associated with the number of true negative variables correctly excluded from the models

\begin{tabular}{ccccc}
\hline Variables & StepAIC & StepBIC & Lasso & Elastic Net \\
\hline npreds & $N S$ & -0.0002 & -0.0259 & -0.0260 \\
noise & -0.0002 & 0.0000 & 0.0110 & 0.0112 \\
typeR2 & -0.0034 & -0.0005 & $N S$ & $N S$ \\
typeR3 & -0.0130 & -0.0039 & -0.0131 & -0.0386 \\
typeR4 & -0.0075 & -0.0023 & -0.0136 & -0.0291 \\
ntrain5000 & 0.0061 & 0.0086 & -0.0349 & -0.0406
\end{tabular}

Table 4. Percentage difference in true effect sizes

\begin{tabular}{cccccc}
\hline Variables & StepAIC & StepBIC & Lasso & Ridge & Elastic Net \\
\hline npreds & 0.0061 & 0.0062 & 0.0066 & 0.0073 & 0.0060 \\
noise & 0.0002 & $N S$ & 0.0055 & 0.0140 & 0.0070 \\
typeR2 & 0.0049 & 0.0027 & $N S$ & 0.0217 & $N S$ \\
typeR3 & 0.0372 & 0.0264 & 0.0533 & 0.1783 & 0.0587 \\
typeR4 & 0.0203 & 0.0136 & 0.0266 & 0.0936 & 0.0295 \\
ntrain5000 & -0.1004 & -0.0859 & -0.2579 & -0.5266 & -0.2861 \\
\hline
\end{tabular}

the effect of the number of noise variables may however be negligible. As expected the specificity with which a noise variable is correctly rejected increases with sample size. Details are shown in Table 3.

When both stepwise and lasso regression algorithms select a true predictor, the correct direction of the effect size is captured accurately (over $99.99 \%$ of the time). Among true predictors that are captured with the right direction in the model, we estimated the percentage difference between the model derived effect sizes and the true effect sizes of the predictors captured in the model. Our results in Table 4 indicate that increasing the total number of variables and increase in correlation between the variables increases the percentage difference between the model derived effect sizes and the corresponding true effect sizes. However, increasing the sample size leads to more precise estimates of the effect sizes. Comparing the effect sizes of the variable selection or model building approach indicate that there may be differences in the magnitude of the effect of the number of noise variables by variable selection or model building algorithm. The shrinkage/regularization algorithms had, on the average, higher effect sizes on the number of variables (true predictors and noise) in the dataset. Or more specifically, higher number of noise variables increase the imprecision with which the effect size is estimated. 


\section{Conclusion}

In this study, we designed and implemented a large scale simulation comparing stepwise model selection using the AIC, stepwise model selection using the BIC criteria, random forest algorithm, lasso, elastic net, and ridge regressions. We varied simulation parameters over a wide range of values that mimic realistic data settings. In particular, we considered 648 distinct simulation scenarios defined by correlation structures for the independent variables, sample sizes, numbers of true predictors and noise variables, as well as small, medium, and large effect sizes in the true model. Regression analysis indicate that the model selection algorithms may be able to take advantage of correlation between variables to improve the AUC even though correlation among variables may lead to false discoveries.

Stepwise model selection using the BIC criteria obtained the highest AUC average over all simulation scenario. All the variable selection algorithms perform well in identifying true predictors but lasso regression suffers most from falsely identifying a noise variable as predictor. Given the parsimonious nature of models resulting from using the BIC as model selection criteria, we expect as obtained that the BIC has the lowest number of noise variables identified as true predictors. Consequently, using the BIC as criteria for model discrimination may result in a parsimonious model as good or better than other model selection algorithms considered in this study. Furthermore, the stepwise model selection by BIC is more likely to identify the true underlying model in terms of the selected predictors. Consequently, we conclude that the stepwise model selection procedures are robust in capturing the true underly relationships and maximizing predictive model performance. 


\section{Abbreviations}

AIC: Aikaike Information Criteria; AICC: Corrected Aikaike Information Criteria; ANOVA: Analysis of Variance; AUC: Area under the receiver operator characteristic curve; BIC: Bayesian Information Criteria; Lasso: Least absolute shrinkage and selection operator

\section{Declarations}

Ethics approval and consent to participate: Not applicable

Consent for publication: Not applicable

Availability of data and materials: This is an empirical study for which details required to replicate over 648,000 datasets have been provided in the manuscript.

Competing interests: The authors do not have any financial or non-financial competing interest to disclose.

\section{Funding: None}

Authors' contributions: LE Conducted the empirical study, interpreted results of the study, wrote draft manuscript, and revised the final manuscript. CR Conceived of the study, provided guidance on each stage of the study/analysis, and revised the draft and final manuscripts.

Acknowledgements: Thanks to Hesham El-Askary, $\mathrm{PhD}$ for his support. 


\section{References}

[1] Esteban MC, Peña JP, Vicente CdVF, Olagorta SG, Álvarez RL, Vicente JdVF. Morbidity and mortality of acute renal failure in the Critical Care Unit of a regional hospital. Revista espanola de anestesiologia y reanimacion. 2018;

[2] Ehwerhemuepha L, Bendig D, Steele C, Rakovski C, Feaster W. The Effect of Malnutrition on the Risk of Unplanned 7-Day Readmission in Pediatrics. Hospital pediatrics. 2018;p. 207-213.

[3] Peacock O, Bassett M, Kuryba A, Walker K, Davies E, Anderson I, et al. Thirty-day mortality in patients undergoing laparotomy for small bowel obstruction. The British journal of surgery. 2018;

[4] Ajay D, Peterson A. MP46-18 A logistic regresion prediction model for the success of the male transobturator sling; valsalva leak-point pressure (VLPP) greater than $70 \mathrm{CM}$ H2O is an indicator for sling success. The Journal of Urology. 2017;197(4):e626-e627.

[5] Flegal KM, Kruszon-Moran D, Carroll MD, Fryar CD, Ogden CL. Trends in obesity among adults in the United States, 2005 to 2014. Jama. 2016;315(21):2284-2291.

[6] Holm DE, Nielen M, Jorritsma R, Irons PC, Thompson PN. Ultrasonographic reproductive tract measures and pelvis measures as predictors of pregnancy failure and anestrus in restricted bred beef heifers. Theriogenology. 2015;

[7] Fan J, Han F, Liu H. Challenges of big data analysis. National science review. 2014;1(2):293-314.

[8] Pirkle JL, Brody DJ, Gunter EW, Kramer RA, Paschal DC, Flegal KM, et al. The decline in blood lead levels in the United States: the National Health and Nutrition Examination Surveys (NHANES). Jama. 1994;272(4):284-291.

[9] Ponce NA, Lavarreda SA, Yen W, Brown ER, DiSogra C, Satter DE. The California Health Interview Survey 2001: translation of a major survey for California's multiethnic population. Public Health Reports. 2004;119(4):388.

[10] Tibshirani R. Regression shrinkage and selection via the lasso. Journal of the Royal Statistical Society Series B (Methodological). 1996;p. 267-288.

[11] Altman DG, Andersen PK. Bootstrap investigation of the stability of a Cox regression model. Statistics in medicine. 1989;8(7):771-783.

[12] Derksen S, Keselman H. Backward, forward and stepwise automated subset selection algorithms: Frequency of obtaining authentic and noise variables. British Journal of Mathematical and Statistical Psychology. 1992;45(2):265-282.

[13] Wilkinson L. Tests of significance in stepwise regression. Psychological Bulletin. 1979;86(1):168.

[14] Harrell FE. Regression modeling strategies. Springer Science \& Business Media; 2001.

[15] Hansen BE. The risk of James-Stein and Lasso shrinkage. Econometric Reviews. 2016;35(8-10):1456-1470.

[16] Tibshirani R. Regression shrinkage and selection via the lasso: a retrospective. Journal of the Royal Statistical Society: Series B (Statistical Methodology). 2011;73(3):273282.

[17] Huang H. Controlling the false discoveries in LASSO. Biometrics. 2017;73(4):11021110.

[18] McLeod A, Xu C. bestglm: Best subset GLM. http://CRANRprojectorg/package=bestglm. 2010;

[19] Ripley B, Venables B, Bates DM, Hornik K, Gebhardt A, Firth D, et al. Package MASS. 2014;. 


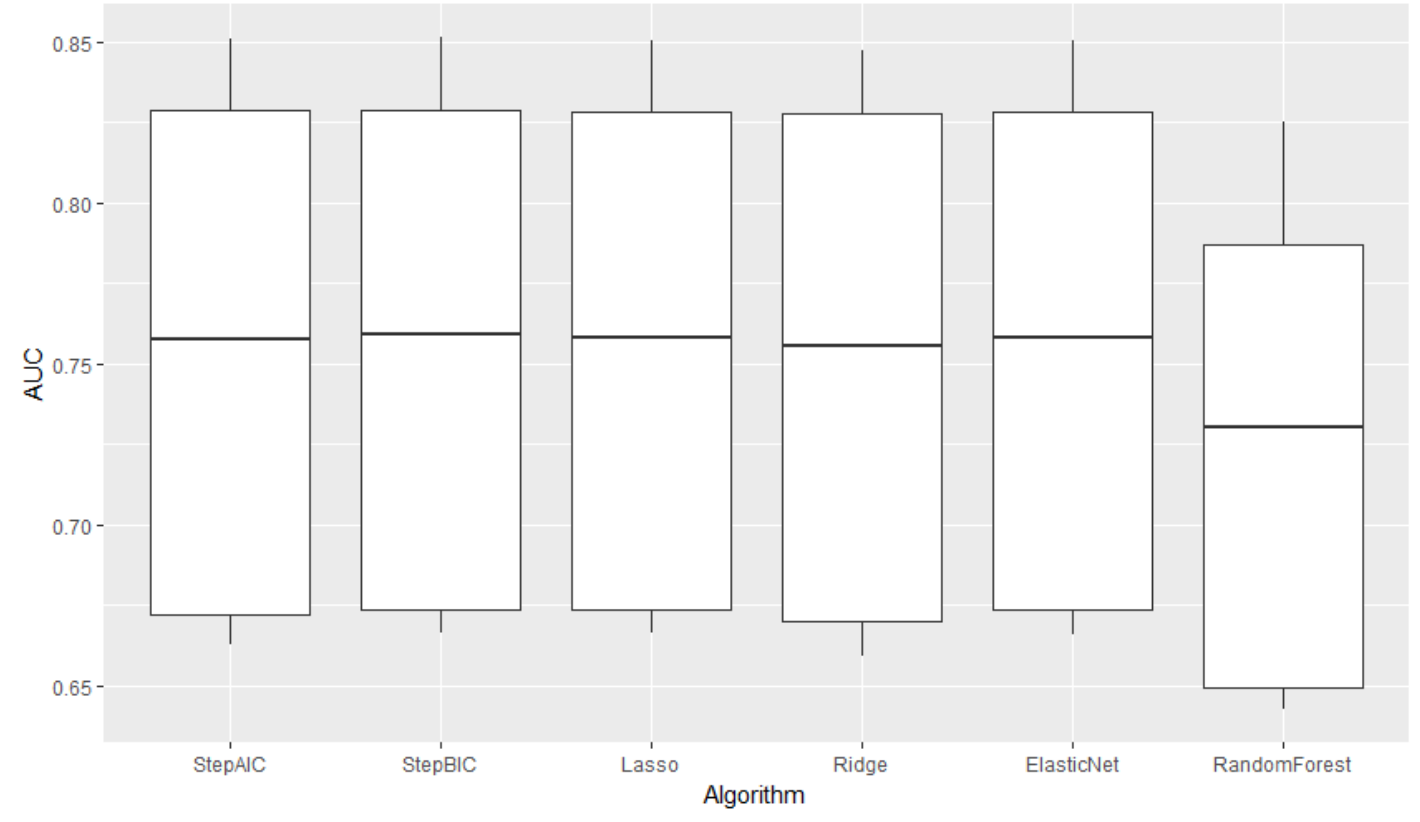

Figure 1. Area under the receiver operator characteristic curve 

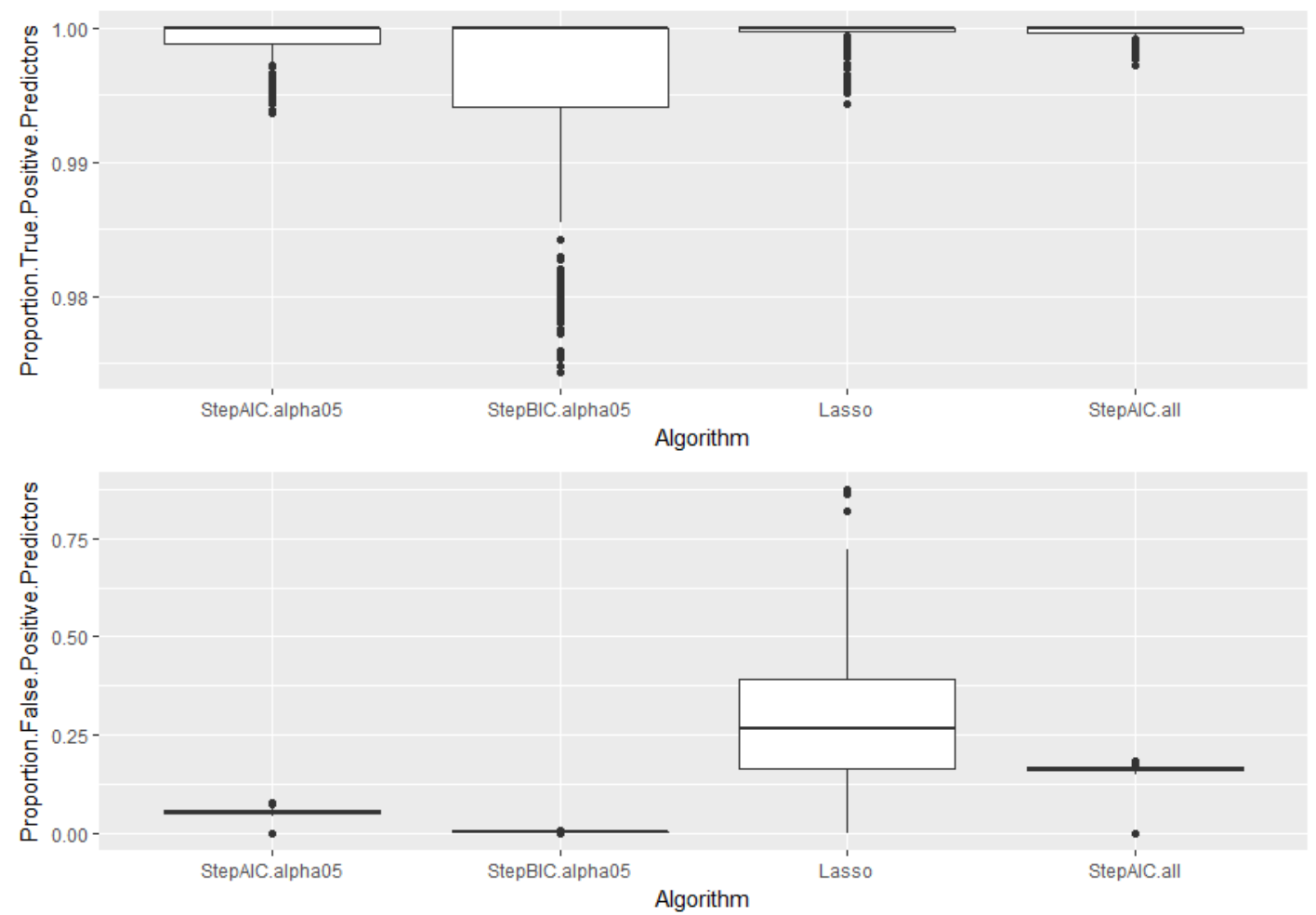

Figure 2. Proportion of true and false positive predictors
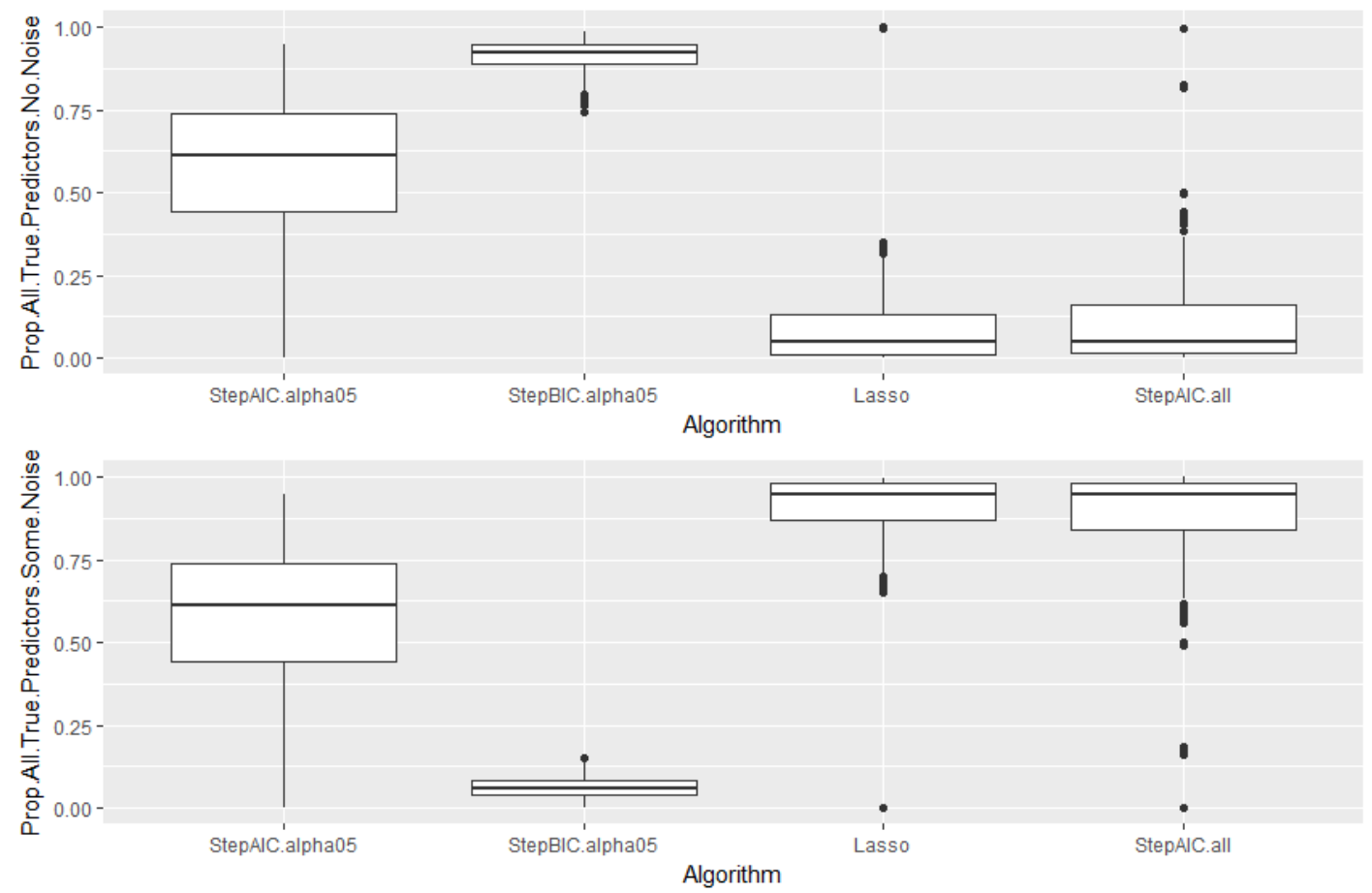

Figure 3. Proportion of times all true predictors were captured with vs without noise variables 

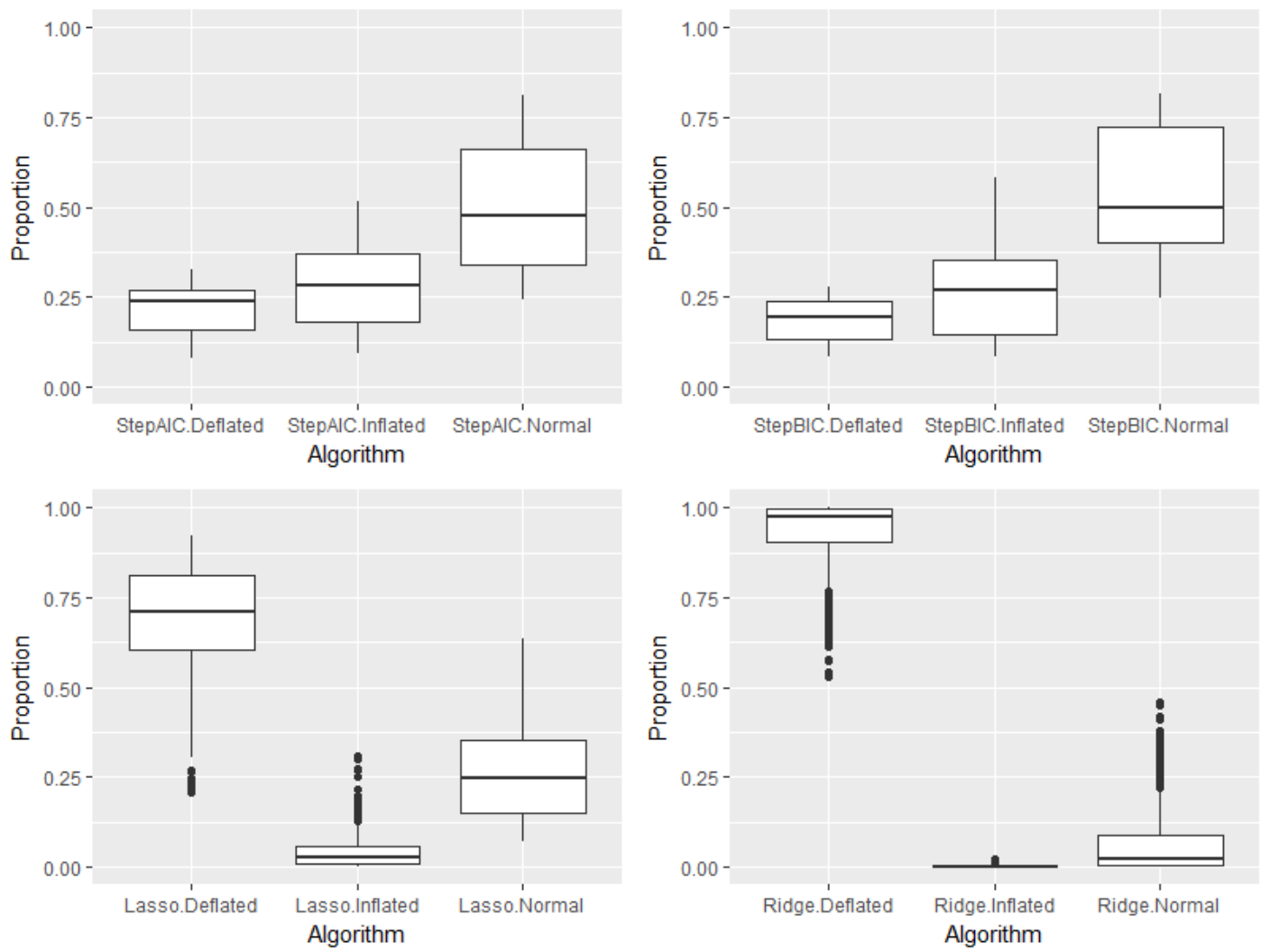

Figure 4. Comparing stepwise regression procedure to Lasso and Ridge regression for precision of effect sizes 